\title{
METILPREDNISOLONA EN BROTES DE ENFERMEDADES INMUNOMEDIADAS. RELACIÓN ENTRE DOSIS, EFECTIVIDAD Y SEGURIDAD. ESTUDIO PILOTO.
}

\author{
METHYLPREDNISOLONE FOR THE TREATMENT OF IMMUNE-MEDIATES DISEASES FLARES. \\ RELATIONSHIP BETWEEN DOSES, EFFECTIVENESS AND SAFETY. A PILOT STUDY.
}

\author{
Ignacio Borgia ${ }^{1}$, Augusto Baccelli², Mabel Goñi ${ }^{3}$, Álvaro Danza ${ }^{4,5}$.
}

\section{Resumen:}

Introducción: Metilprednisolona (MTP) es una molécula con probada efectividad antiinflamatoria e inmunosupresora para la terapia en pulsos de brotes de enfermedades inmunomediadas (EI). Sin embargo, no se conoce con certeza la mejor relación entre dosis, efectividad y efectos adversos.

OBJETIVO: Conocer los usos de MTP, dosis, respuesta terapéutica y efectos adversos.

Material y métodos: Se realizó un estudio retrospectivo de pacientes que recibieron MTP en el Hospital Pasteur de Uruguay. Se incluyeron pacientes que recibieron MTP en pulsos por una enfermedad inmunomediada. Se analizaron las siguientes variables: edad, sexo, dosis acumulada de MTP, duración del tratamiento con MTP, respuesta terapéutica, efectos adversos.

Resultados: Se identificaron 36 casos, 27 de sexo femenino. La media de edad fue 41,5 (DE: 15,5) años. La media de dosis en los casos de respuesta completa fue 3,6 (DE: 2) gramos; la media de dosis en los casos de respuesta parcial fue 4 (DE: 1,5) gramos; la media de dosis de no respuesta fue 2,8 (DE 1,2) gramos $(p>0,05)$. La media de dosis en los pacientes que presentaron efectos adversos fue 4,4 (DE: 1,8$)$ gramos y la media de dosis de los que no presentaron efectos adversos fue $3,2(\mathrm{DE}: 1,6)$ gramos $(p<0,05)$.

Conclusión: Nuestra serie sugiere que no existe una clara relación entre dosis mayores y mejores respuestas terapéuticas. Sin embargo, existe una mayor tendencia a presentar efectos adversos con dosis más elevadas. Estos hallazgos refuerzan la necesidad de racionalizar las dosis de MTP y continuar estudiando este tema en con mayor número de pacientes.

Palabras clave: metilprednisolona; efectividad; efectos colaterales y reacciones adversas relacionados con medicamentos; relación dosis-respuesta a droga.

\section{Abstract:}

Background: Methylprednisolone (MTP) pulses have proved effectiveness for the treatment of severe manifestations of immune-mediated diseases (ID). However, the the relationship between doses, effectiveness and safety is not well established.

Objective: To know MTP indications, doses, therapeutic response and safety in the clinical setting.

Methodology: A retrospective review of records was performed in Hospital Pasteur of Uruguay, from patients who had received MTP during a ID flare. Variables studied: age, sex, MTP cumulative doses, duration of treatment, therapeutic response and adverse effects.

Results: Thirty six cases were identified, 26 were female. The mean age was 41,5 (SD 15,5) years. The mean dose of MTP in cases of complete response was 3,6 (SD 2) grams, the mean dose in cases of partial response was 4 (SD 1,5) grams, and in cases of no response was 2,8 (SD 1,2) grams ( $p>0,05)$. The mean dose in patients with adverse effects was 4,4 (SD 1,8) grams, and in cases without adverse effects was 3,2 (SD 1,6) grams $(p<0,05)$.

Conclusions: Our series does not confirm a relationship between higher doses and clinical response. However, an association between higher doses of MTP and adverse effects was observed. These finding should be thoroughly investigated.

Keywords: methylprednisolone; effectiveness; drug-related side effects and adverse reactions; dose-response relationship. drug

\footnotetext{
${ }^{1}$ Asistente de Clínica Médica. Grupo de Enfermedades Autoinmunes Sistémicas. Clínica Médica - Hospital Pasteur. Facultad de Medicina, Universidad de la República - Administración de Servicios de Salud del Estado. Uruguay.

${ }^{2}$ Residente Asistente de Clínica Médica. Grupo de Enfermedades Autoinmunes Sistémicas. Clínica Médica - Hospital Pasteur. Facultad de Medicina, Universidad de la República - Administración de Servicios de Salud del Estado. Uruguay.

${ }_{3}$ Profesora de Clínica Médica. Grupo de Enfermedades Autoinmunes Sistémicas. Clínica Médica - Hospital Pasteur. Facultad de Medicina, Universidad de la República - Administración de Servicios de Salud del Estado. Uruguay.

${ }^{4}$ Profesor Agregado de Clínica Médica. Grupo de Enfermedades Autoinmunes Sistémicas. Clínica Médica - Hospital Pasteur. Facultad de

Medicina, Universidad de la República - Administración de Servicios de Salud del Estado. Uruguay

${ }^{5}$ Email de contacto: alvarodanza@gmail.com
} 


\section{Introducción}

Los glucocorticoides (GC) son fármacos ampliamente utilizados en la práctica clínica para el tratamiento de múltiples enfermedades inmunomediadas (EI). Se caracterizan por presentar un potente efecto antiinflamatorio e inmunosupresor. Se ha acumulado importante experiencia en su uso, en base al número de pacientes tratados y a las diversas entidades en las que han sido empleados. Sin embargo, aún existen numerosas incertidumbres en relación a las dosis más razonables, los tiempos de administración más adecuados y los planes de descenso más aconsejables.

En los últimos años se ha comprobado que actúan básicamente por dos vías de acción: la vía genómica y la no genómica ${ }^{(1)}$. La primera de ellas, ampliamente estudiada y conocida, se caracteriza por la unión de la molécula de GC a un receptor citoplasmático. Este complejo receptor-ligando se trasloca al núcleo celular donde se une a secuencias específicas de ADN. A este nivel el complejo inhibe la expresión de genes que están involucrados en la respuesta inflamatoria, pero también estimula la expresión de genes que están implicados con los efectos adversos de los GC. Dentro de las primeras, se destaca la reducción de moléculas de adhesión así como también la interferencia en la función de leucocitos, fibroblastos y células endoteliales $^{(2)}$. Este efecto es potencialmente saturable, no habiéndose comprobado un aumento marcado de su efecto por encima de dosis 30 de $\mathrm{mg}$ de prednisona o equivalente. Los efectos generados por dicho mecanismo se inician de forma lenta, requiriéndose al menos 30 minutos para su inicio, y por los menos 4 a 6 horas para alcanzar la totalidad de su efecto. Se destaca que estos efectos pueden desencadenarse incluso a dosis menores a 30 $\mathrm{mg}$ de prednisona $\mathrm{o}$ equivalente. $\mathrm{La}$ particularidad de esta vía es que el receptor citosólico está expresado en todas las células del organismo, lo que explica que los efectos adversos sean tan variados, afectando a prácticamente todos los tejidos ${ }^{(3)}$.

La vía no genómica se activa frente a dosis mayores $^{(1-3)}$. Se caracteriza por modular un receptor de membrana y vías moleculares intracelulares diferentes a las de la vía genómica. Se postula que esta vía se activa a dosis por superiores a los $100 \mathrm{mg}$ de prednisona 0 equivalentes. El inicio de acción de estos efectos es más corto (segundos a minutos) y su potencia antiinflamatoria, en el caso de algunos GC, como Metilprednisolona (MTP) y Dexametasona, es hasta cinco veces mayor que por la vía genómica. Esto avala el uso de estos medicamentos a dosis mayores, en forma de pulsos, en particular en el caso de brotes graves o resistentes de enfermedades inmunomediadas $^{(4,5)}$.

Los efectos adversos de los GC son múltiples y conocidos. Está bien establecido que existe una relación lineal entre la dosis y la duración del tratamiento y la aparición de ciertos efectos adversos $^{(6)}$. A nivel óseo se destaca la osteoporosis y la eventualidad de osteonecrosis aséptica. A nivel cardiovascular, la Hipertensión Arterial y la dislipidemia con el consiguiente aumento del riesgo cardiovascular absoluto $y$ mayor incidencia de enfermedad coronaria. A nivel endocrinológico, se destaca la intolerancia a la glucosa, diabetes, redistribución de la grasa corporal y aumento de peso y la supresión de las hormonas sexuales, pudiendo determinar el conocido Síndrome de Cushing. A nivel oftalmológico se vinculan con la aparición de cataratas y aumento de la presión intraocular. También se ha relacionado el uso de GC con efectos adversos a nivel gastrointestinal, en particular con úlcera gastroduodenal, si bien su frecuencia parece ser baja ${ }^{(7)}$.

Las infecciones merecen una mención aparte dada la importante morbimortalidad que generan. En la Cohorte EUROLUPUS, de 1000 pacientes con lupus eritematoso sistémico (LES), 36\% sufrieron infecciones ${ }^{(8)}$. Además, en esa cohorte, un $25 \%$ de las muertes fueron atribuidas a infecciones. Se ha establecido con certeza que el uso de GC aumenta la susceptibilidad a todo tipo de infecciones, bacterianas, virales, fúngicas y parasitarias, siendo las primeras las más frecuentes $^{(9)}$. El riesgo de infección aumenta con la dosis y duración del tratamiento y tiende a mantenerse baja en pacientes tratados con dosis bajas de GC, en particular por debajo de 7,5 $\mathrm{mg} /$ día de prednisona o equivalentes ${ }^{(10)}$. Al igual que ocurre con el uso prednisona, se ha sugerido que el uso de pulsos de MTP por encima de 1,5 gramos aumenta el riesgo de infecciones severas $^{(11)}$.

No existe claro consenso respecto a las dosis ni al tiempo de administración de GC a utilizar ${ }^{(12)}$. Esto ocurre tanto para el caso de prednisona, como para los pulsos de $\mathrm{MTP}^{(13)}$. No hay estudios concluyentes que comparen diferentes dosis de GC, siendo la mayoría no controlados y retrospectivos. Evidencias recientes sugieren que dosis de $30 \mathrm{mg}$ por día de prednisona o equivalente aportan beneficios terapéuticos comparables a los de los clásicos esquemas de 1 $\mathrm{mg} / \mathrm{kg} / \mathrm{día}$, determinando claramente menores efectos adversos ${ }^{(14)}$. Con el uso de MTP existen dudas similares, no se conoce la dosis en la cual 
la relación beneficio/riesgo es óptima ${ }^{(13-15)}$. En consecuencia, tanto la dosis de prednisona de 1 $\mathrm{mg} / \mathrm{kg} /$ día como los pulsos de MTP de 1 gramo por día, son esencialmente empíricos, no basados en evidencia sólida.

Metilprednisolona (MTP) es una molécula con probada efectividad antiinflamatoria e inmunosupresora. La terapia en pulsos es ampliamente utilizada para inducir la remisión de brotes graves de El. Si bien su uso es extenso, no se conoce con certeza la mejor relación entre dosis, efectividad y efectos adversos. El objetivo de este estudio es conocer el perfil de uso de la MTP en un Hospital general de agudos, describiendo las patologías en las que se emplea y evaluar la relación existente entre las dosis empleadas, respuesta terapéutica y efectos adversos.

\section{Material y Métodos}

Se diseñó un estudio piloto, retrospectivo y analítico. Se realizó un muestreo por conveniencia, incluyendo todos los pacientes que recibieron pulsos de MTP intravenosa entre 1 de enero de 2013 y el 31 de diciembre de 2015 por una El en el Hospital Pasteur. El Hospital Pasteur es un Hospital general, de adultos, de segundo y tercer nivel asistencial, de internación para patologías agudas. El Servicio de Medicina Interna cuenta con 110 camas de internación y en él funciona de forma integrada realizando actividades asistenciales y académicas una Clínica Médica de la Facultad de Medicina.

Siguiendo las recomendaciones, se definió pulso de MTP al uso a dosis mayores a 100 mg por vía intravenosa. Se establecieron las siguientes categorías de El: neuroinmunomediada, enfermedades autoinmunes sistémicas (EAS), hematológica (anemia hemolítica autoinmune y trombocitopenia inmune), nefrológica y gastrointestinal. Los datos fueron obtenidos del registro de la Farmacia Hospitalaria. Con estos datos, se analizaron las historias clínicas de los pacientes. A los efectos de recabar los datos se confeccionó un formulario pre-codificado de recolección de datos.

Las comorbilidades fueron agrupadas en: Diabetes, enfermedad cardiovascular, tabaquismo, infección por el virus de la inmunodeficiencia humana (HIV), enfermedad respiratoria y enfermedad renal crónica $(E R C)$.

Se analizaron las siguientes variables: edad, sexo, patología que motivó el uso de MTP, comorbilidades relevantes, dosis acumulada de MTP, días de internación, duración del tratamiento con MTP, uso de Prednisona posterior al uso de MTP, otros inmunosupresores indicados, efectos adversos,

respuesta

(completa, parcial o no respuesta).

La respuesta al tratamiento se evaluó en función de la clínica y los parámetros inflamatorios así como parámetros específicos de cada patología. A tales efectos se consideran las siguientes respuestas: completa, parcial y no repuesta:

- Respuesta completa: evolución a la mejoría clínica franca de los síntomas que motivaron la indicación del tratamiento, objetivado esto por el médico tratante. Además, mejoría de más $50 \%$ de al menos uno de los parámetros inflamatorios convencionales (VES, PCR, complemento, títulos de Anti-DNA, o lo que se empleara como parámetro analítico de control evolutivo).

- Respuesta parcial: evolución a la mejoría parcial 0 incompleta de los síntomas que motivaron la indicación del tratamiento, objetivado esto por el médico tratante. Se asocia con una mejoría entre $25 \%$ y $50 \%$ de al menos uno de los parámetros inflamatorios convencionales.

- No respuesta: mantenimiento o peoría clínica de los síntomas, además, no descenso de los parámetros inflamatorios en los valores indicados previamente o peoría de los mismos.

Los efectos adversos se registran de forma dicotómica: presentes 0 ausentes. Las infecciones se clasificaron en: ausente, severa y no severa. Se definió como severa aquella que provocó bacteriemia, requirió ingreso a cuidados especializados, prolongó la estadía hospitalaria o determinó ingreso nosocomial.

En todo el proceso se mantuvo el anonimato de los pacientes, respetando los principios establecidos en la Declaración de Helsinki para la investigación biomédica. Se obtuvo autorización de las autoridades del Hospital Pasteur.

Los datos descriptivos de las variables de distribución normal se presentan con la media y el desvío estándar (DE), los datos cualitativos se expresan en frecuencias y porcentajes. Para comparar las medias se utilizó la prueba de t de Student, con un nivel de significancia del 5\% (alfa $0.05)$. Los datos se procesarán en el programa SPSS (versión 18.0).

\section{Resultados}

Se identificaron 36 casos de pacientes que recibieron MTP por una El, $27(75 \%)$ de sexo femenino. La media de edad fue 41,5 (DE: 15,5) años. El promedio de días de internación en los casos identificados que recibieron MTP fue 21 (DE: 18) días. Las enfermedades en las que se empleó fueron: EAS 13 (36\%), enfermedad 
neuroinmunomediada 13 (36\%), hematológica 4 $(11,1 \%)$ nefrológica y gastrointestinal $6(16,7 \%)$. Las comorbilidades asociadas fueron: diabetes en $8(22 \%)$ casos, enfermedad cardiovascular 14 (38\%), tabaquismo $4(11,1 \%)$, enfermedad respiratoria crónica $3(8,5 \%)$, ERC 3 (8,3\%), HIV $1(2,7 \%)$ caso. (Tabla 1$)$.

Tabla 1: Características basales de la población incluida.

\begin{tabular}{|l|l|}
\hline Sexo femenino (n, \%) & $27(75 \%)$ \\
\hline Edad (años) (media y DE) & $41,5(\mathrm{DE} 15,5)$ \\
\hline Días internación (media y DE) & $21(\mathrm{DE} 18)$ \\
\hline Enfermedad inmunomediada & $\mathbf{n}(\%)$ \\
\hline EAS & $13(36)$ \\
\hline Neuroinmunomediada & $13(36)$ \\
\hline Hematológica & $4(11,1)$ \\
\hline Nefrológica y gastrointestinal & $6(16,7)$ \\
\hline Comorbilidades & $\mathbf{n ~ ( \% )}$ \\
\hline Diabetes mellitus & $8(22)$ \\
\hline Enfermedad cardiovascular & $14(38)$ \\
\hline Tabaquismo & $4(11,1)$ \\
\hline Respiratoria & $3(8,3)$ \\
\hline VIH & $1(2,7)$ \\
\hline Enfermedad renal crónica & $3(8,3)$ \\
\hline
\end{tabular}

La media de dosis acumulada fueron 3,6 (DE: $1,7)$ gramos, y la dosis máxima fueron 8 gramos, registrada en un único caso. La media de duración de tratamiento con MTP fue 4 (DE: 1,5) días. Diecinueve $(52,8 \%)$ pacientes presentaron respuesta completa, $11(30,6 \%)$ respuesta parcial y $6(16,7 \%)$ no tuvieron respuesta.

Veintisiete pacientes $(75 \%)$ recibieron prednisona luego de los pulsos de MTP. La media de dosis acumulada a las 4 semanas de prednisona fue 1085 (DE: 420) gramos, la dosis máxima fue de $60 \mathrm{mg}$ por día y la medida diaria en 4 semanas fue $40 \mathrm{mg}$. Se empleó otro inmunosupresor adicional en 16 (44\%) de los casos. Se empleó concomitantemente prednisona con un inmunosupresor en 14 (52\%) de los casos.

La media de dosis en los casos de respuesta completa fue 3,6 (DE: 2) gramos; la media de dosis en los casos de respuesta parcial fue 4 (DE: 1,5) gramos; la media de dosis de no respuesta fue 2,8 (DE 1,2) gramos ( $p=N S)$ (tabla 2, gráfico 1).

Tabla 2: Respuesta y dosis de Metilprednisolona.

\begin{tabular}{|l|l|l|}
\hline RESPUESTA & DOSIS MTP (g) & \\
\cline { 1 - 2 } Completa & $3,6(2)$ & \multirow{2}{*}{$\mathrm{p}>0,05$} \\
\hline Parcial & $4(1,5)$ & \\
\hline No respuesta & $2,8(1,2)$ \\
\hline
\end{tabular}

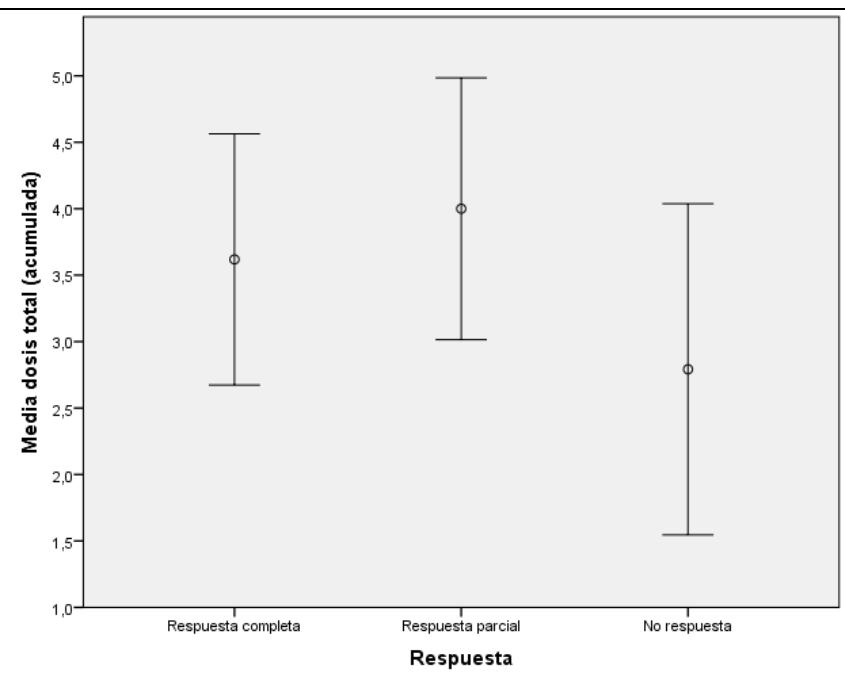

Gráfico 1. Se muestra la media de dosis de MTP con el Intervalo de Confianza $95 \%$

de acuerdo al tipo de respuesta (completa, parcial, no respuesta), $p>0,05$

Se constataron 12 efectos adversos (33,3\%), 8 $(22,2 \%)$ infecciones, 4 severas. Dentro de los pacientes con comorbilidades, 6 de los 8 pacientes con Diabetes presentaron efectos adversos respecto a los pacientes con comorbilidades diferentes a la diabetes (6/20), $\mathrm{p}<0,05$ (gráfico 2).

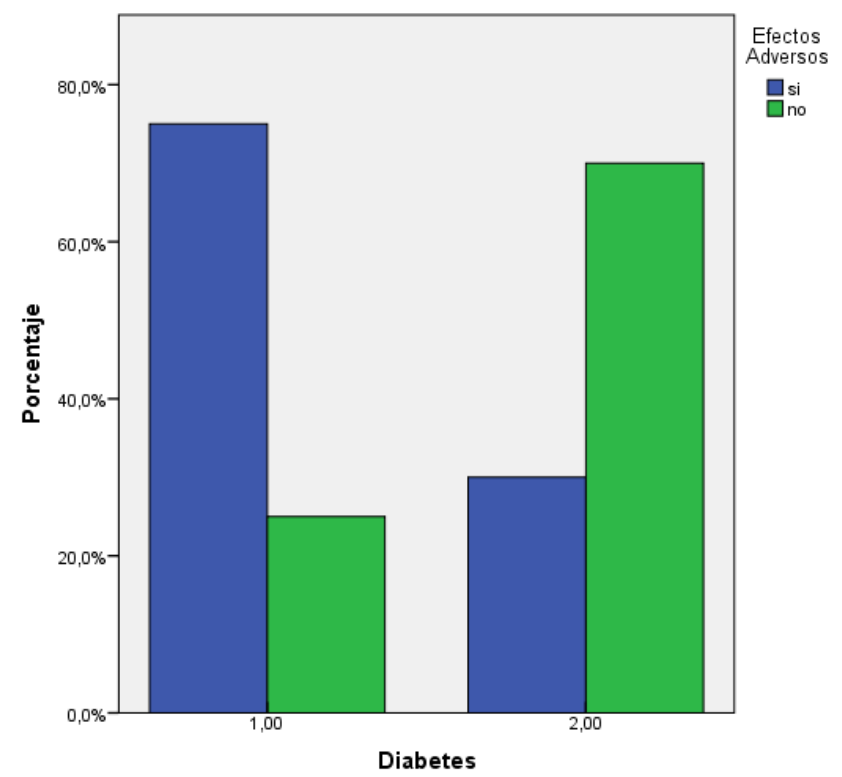

Gráfico 2. Se presentan la presencia de efectos adversos en relación a la presencia o no de Diabetes Mellitus. Nota: $1=$ presencia de Diabetes; $2=$ ausencia de Diabetes. $(p<0,05)$.

La media de dosis en los pacientes que presentaron efectos adversos fue 4,4 (DE: 1,8) gramos y la media de dosis de los que no presentaron efectos adversos fue 3,2 (DE: 1,6) gramos $(p<0,05)$ (gráfico 3$)$. No se objetivaron diferencias estadísticamente significativas entre las infecciones severas y la dosis de MTP $(p=N S)$. 


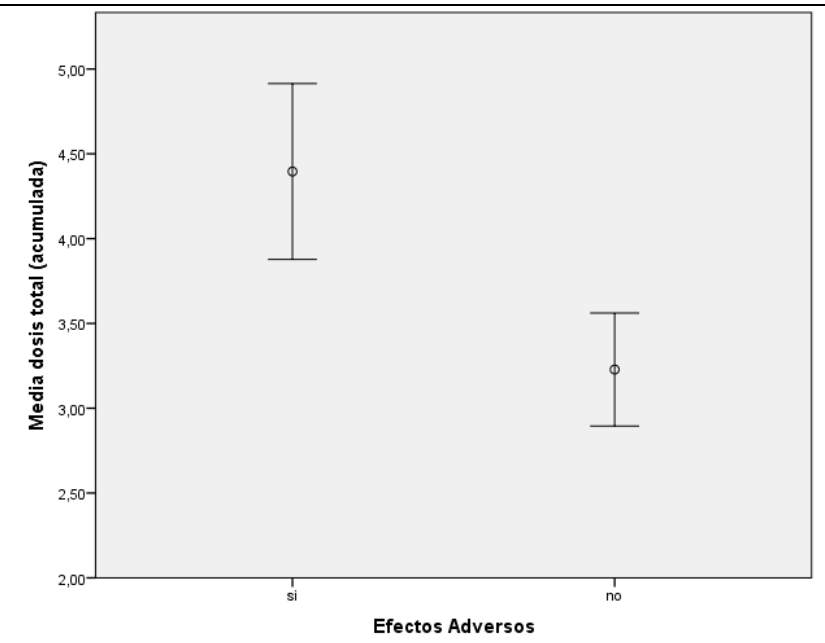

Gráfico 3. Se presenta la media de dosis acumulada a la que aparecen efectos adversos $+/-1$ error estándar $(p<0,05)$.

\section{Discusión}

El presente estudio muestra una importante dispersión tanto en relación a las dosis empleadas de MTP como a la duración de la terapia en pulsos. Esto confirma que si bien hay recomendaciones generales, en la práctica clínica se trata de un tema claramente no sistematizado. Nuestros datos muestran que se emplea MTP en diversas enfermedades inmunomediadas, por lo que se trata de un recurso terapéutico que debe ser conocido por los clínicos que asisten pacientes con este tipo de patologías. El perfil de comorbilidades está acorde con los usuarios que se asisten en nuestro Centro y con las patologías más prevalentes de la población general del Uruguay ${ }^{(16)}$.

El uso de MTP predominó en pacientes jóvenes y de sexo femenino, compatible con el tipo de enfermedades en las que se la utilizó, habida cuenta del predominio de éstas en este grupo de pacientes. El promedio de días de internación está claramente por encima del promedio de nuestro Hospital (10 días). Este dato es esperable en el contexto de brotes severos de enfermedades complejas pero aun así, podría reducirse utilizando esquemas terapéuticos más acotados en tiempos y dosis, reduciendo de este modo los costos asistenciales.

Los datos muestran que el uso de MTP determina respuestas favorables en la mayoría de los pacientes analizados. Sin embargo, nuestros datos sugieren que no existe clara relación entre la respuesta terapéutica y las dosis utilizadas de MTP. Este hallazgo es compatible con datos presentados por otros autores, que han comprobado que dosis mayores no determinarían mejores respuestas clínicas ${ }^{(11,17)}$. A su vez, es compatible con el perfil farmacológico de este medicamento. Tal como se menciona más arriba, no se ha comprobado que dosis mayores de GC sean más efectivas, ni en el caso de la vía genómica ni en el caso de la vía no genómica. En efecto, es probable que ambas vías sean saturables, no conociéndose aún la dosis exacta de saturación $n^{(12,18,19)}$.

En la mayoría de los pacientes se empleó Prednisona a continuación de los pulsos de MTP a dosis que actualmente se definen como altas. Llama la atención que en El, menos de la mitad de los casos reciben otro inmunosupresor adicional como ahorrador de corticoides, lo que denota ciertas dificultades en el uso de este grupo de medicamentos. Es posible que el equipo médico esté poco familiarizado con estos medicamentos y más con los GC por lo que estos últimos se usan en mayor medida. Sin embargo, el potencial de efectos adversos exige el empleo precoz de otros inmunosupresores como ahorradores de corticoides, de forma tal de reducir las dosis y duración del tratamiento con estos últimos, disminuyendo así el riesgo de efectos adversos que estos determinan.

En este estudio, un tercio de los pacientes presentaron efectos adversos, que aparecieron con mayor frecuencia a dosis más altas. Esto no llama la atención, en la medida que, como se menciona previamente, los efectos adversos de los GC son dosis y tiempo dependientes. Es interesante destacar que los efectos adversos fueron más frecuentes en diabéticos y que las infecciones fueron los efectos adversos más frecuentes. Es posible que otros efectos adversos se observen en plazos mayores, lo que claramente estuvo por fuera del alcance de este trabajo.

En línea con nuestras observaciones, un estudio retrospectivo realizado en Singapur en pacientes con LES comparó la respuesta terapéutica y los efectos adversos de dos grupos de pacientes que recibieron MTP en el contexto de brotes severos. El primer grupo estuvo constituido por 26 que recibieron dosis entre 1 y 1,5 gramos de MTP ("bajas dosis"), el segundo por 29 pacientes que recibieron dosis entre 3 y 5 gramos ("dosis altas"). Los dos grupos presentaron una respuesta terapéutica similar pero el grupo que recibió dosis bajas presentó menor frecuencia de efectos adversos, sobre todo infecciosos ${ }^{(11,15)}$.

En base a estas observaciones y otras que hemos desarrollado, nuestro grupo postula un esquema terapéutico que está siendo probado en estudios clínicos en desarrollo. En particular, nuestro enfoque consiste en usar la terapia en pulsos para casos moderados a severos, no superando la dosis de $500 \mathrm{mg}$ por día en tres días consecutivos. En casos de brotes 
moderados recurrimos a dosis incluso menores, de $250 \mathrm{mg}$ por día por tres días consecutivos. Hemos abandonado las dosis de 3 gramos y periodos más prolongados de administración. Finalmente, en caso de requerirse mantener el tratamiento glucocorticoideo, la do sis máxima que empleamos son $30 \mathrm{mg}$ por día por no más de 30 días. Esto se acompaña, necesariamente, del agregado precoz de un inmunosupresor de mantenimiento ${ }^{(14,20,21)}$.

Nuestro trabajo tiene limitaciones dadas por el número reducido de casos y el diseño retrospectivo. Dentro de las fortalezas destaca que es una aproximación en base a casos reales, locales y de uso de este medicamento en un Hospital general de adultos. Estos datos son relevantes a la hora de evaluar la efectividad de los medicamentos, término que refiere a los beneficios que los medicamentos determinan en condiciones reales de uso, bien diferentes a las condiciones ideales de los ensayos clínicos ${ }^{(22)}$. Vale aclarar que no se dispone de ensayos clínicos que confirmen cuáles son las dosis ni la duración óptima del tratamiento con GC. Esto aplica para la terapia en pulsos y para la terapia prolongada.

Nuestro trabajo aporta datos relevantes en relación al uso de MTP, sugiriendo que no hay una clara relación entre dosis y respuesta clínica pero sí entre las dosis y los efectos adversos. Estos resultados deberían servir de estímulo para generar nuevos estudios prospectivos, comparando, eventualmente, dos esquemas con dosis diferentes. De este modo, se podrán desarrollar guías y pautas que permitan racionalizar las dosis de MTP, obteniendo la mejor relación dosis-efectividad-seguridad.

\section{Bibliografía}

1. Buttgereit F, Wheling M, Burmester GR. A New Hypothesis of Modular Glucocorticoid Actions. Arthritis Rheum 1998; 41: 761-7.

2. Buttgereit F, Burmester GR, Lipworth BJ. Optimised glucocorticoid therapy: the sharpening of an old spear. Lancet 2005; 365: 801-03.

3. Rhen T, Cidlowski JA. N Engl J Med 2005; 353: 171123.

4. Lipworth BJ. Therapeutic implications of non-genomic glucocorticoid activity. Lancet 2000; 356: 87-9.

5. Buttgereit $F$, da Silva JA, Boers M, Burmester GR, Cutolo $M$, et al. Standardized Nomenclature for Glucocorticoids Dosages and Glucocorticoid Treatment regimens: current questions and tentative answers in rheumatology. Ann Rheum Dis 2002; 61: 718-22.

6. Ruiz-Irastorza G, Danza A, Khamashta M. Use and Abuse in SLE. Rheumatology 2012; 51: 1145-53.

7. Da Silva JA, Jacobs JW, Kirwan JR, Boers M, Saag $K G$, et al. Safety of Low Dose Glucocorticoids Treatment in Rheumatoid Arthritis: published evidence and prospective trial data. Ann Rheum Dis 2006; 65: 285-93.

8. Cervera R, Kamashta MA, Font J, Sebastiani G D, Gil $A$, et al . Morbidity and mortality in systemic lupus erythematosus during a 10 year period. Medicine (Baltimore) 2003; 82: 299-308.

9. Danza A, Ruiz-Irastorza G. Infection risk in systemic lupus erythematosus patients: susceptibility factors and preventive strategies. Lupus 2013; 22: 1286-94.

10. Ruiz-Irastorza G, Olivares N, Ruiz-Arruza I, MartínezBerriotxoa A, Egurbide MV, et al. Predictors of major infections in systemic lupus erythematosus. Arthritis Res Ther 2009; 11:R109.

11. Badsha H, Kong KO, Lian TY, Chan SP, Edwards CJ, et al. Low-dose pulse methylprednisolone for systemic lupus eritematosus flares is efficacious and has decreased risk of infectious complications. Lupus 2002; 11: 508-13.

12. Buttgereit $F$, Straub $R H$, Wheling $M$, Burmester GR. Glucocorticoids in the treatment of rheumatic diseases: An update on the mechanisms of action. Arthritis Rheum 2004; 50: 3408-17.

13. Franchin G, Diamond B. Pulse Steroids: How Much Is Enough? Autoimmun Rev 2006; 5: 111-13.

14. Ruiz-Irastorza G, Danza A, Perales I, Villar I, García M, et al. Prednisone in lupus nephritis: how much is enough? Autoimmun Rev 2014; 13: 206-14.

15. Badsha $H$, Edwards CJ. Intravenous pulses of methylprednisolone for systemic lupus erythematosus. Sem Arthritis Rheum 2003; 32: 370-7.

16. Cuadro mortalidad por causa, sexo y edad en Uruguay, año 2015. www.msp.gub.uy/poblacion/estadisticas-demortalidad. Consultado el 11 de enero de 2017.

17. Edwards JC, Snaith ML, Isenberg DA. A double blind controlled trial of methylprednisolone infusions in systemic lupus erythematosus using individualised outcome assessment. Ann Rheum Dis 1987; 46: 773-6.

18. Stahn C, Buttgereit F. Genomic and nongenomic effects of glucocorticoids. Nat Clin Pract Rheumatol 2008; 4: 525-33

19. Van der Goes MC, Strehl C, Buttgereit F, Bijlsma JW, Jacobs JW. Can adverse effects of glucocorticoid therapy be prevented and treated? Expert Opin on Pharmacother 2016; 17: 2129-33

20. Ruiz-Irastorza G, Danza A, Kamashta M. Tratamiento del lupus eritematoso sistémico: mitos, certezas $y$ dudas. Med Clin (Barc) 2013; 21: 533-42

21. Conde JL. Eficacia y efectividad: una distinción útil para la práctica y la investigación clínica. Nefrología 2002; 22: 219-22.

22. Strehl C, Buttgereit F. Optimized glucocorticoid therapy: Teaching old drugs new tricks. Mol Cell Endocrinol 2013; 380: 32-40 